\title{
Incidence of surgical site infections after caesarean sections in a community hospital
}

Tyler Rouse, MD, FRCP $(\mathrm{C}) ;{ }^{1}$ Patricia Nascu, MD, FRCS(C); ${ }^{2}$ Charlotte Dawson, MD; ${ }^{3}$ Emily Morris $^{4}$

${ }^{\prime}$ Associate Pathologist, Huron Perth Healthcare Alliance; Adjunct Professor, Department of Pathology, Schulich School of Medicine, Western University, London, ON, Canada ${ }^{2}$ Adjunct Professor, Department of Obstetrics and Gynecology, Schulich School of Medicine, Western University, London, ON, Canada

${ }^{3}$ Postgraduate training, Department of Family Medicine, Queen's University, Kingston, ON, Canada

${ }^{4}$ Undergraduate student, University of Toronto, Toronto, ON, Canada

\section{Corresponding author:}

Dr. Tyler Rouse, Associate Pathologist

Huron Perth Healthcare Alliance

46 General Hospital Dr.

Stratford, ON N5A 2Y6 Canada

Tel. (office): $519-272-8210 \times 2873$

tyler.rouse@hpha.ca

\section{ABSTRACT}

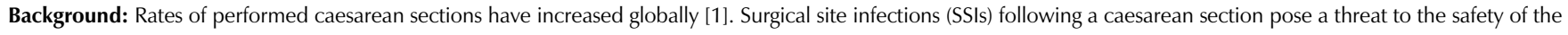
patient. This study intended to determine the current SSI rate after caesarean sections at one community hospital. The rate of incidence of these infections was compared to benchmark rates from various studies, including a report from the American National Healthcare Safety Network (NHSN). This comparative study provides objective evidence of performance in relation to SSIs.

Method: The primary data collection method included a form completed by the obstetrician-gynecologists of the individual patients at the six-week post-partum follow-up visit. Demographic data was collected retrospectively through analysis of medical records. Patients who underwent a caesarean section in the seven-month data collection period (between November 2015 and May 2016) were asked to participate, and consent was obtained.

Results: A total of 118 caesarean sections were reviewed and seven SSIs diagnosed. A crude SSI rate was calculated at 5.9\%. For further insight, NHSN risk-adjusted SSI rates were calculated. The NHSN risk-adjusted SSI rate was determined at $6.1 \%$ for those patients presenting with a risk index level of 0 and at $5.9 \%$ for those with a risk index level of 1 . Both NHSN risk index levels of 2 and 3 were identified to have an adjusted SSI rate of $0.0 \%$

Conclusion: This study, while limited in scope, does add to the collective literature on SSI rates following caesarean sections. Most significantly, it provides a methodology for other centres interested in determining their own infection rates and could lead to improved practices and better patient outcomes.

\section{KEYWORDS}

Surgical site infection rate; caesarean section; community hospital

\section{INTRODUCTION}

Healthcare infections can contribute largely to the morbidity and mortality of hospitalized patients. This study examined post-caesarean section surgical site infection (SSI) rates at a community hospital and compared these rates to other reported benchmarks. Globally, the rate of caesarean sections performed has increased [1]. In Canada, the 2016-2017 rate of caesarean sections performed was $28.2 \%$ of births, an increase from the previous $26.7 \%$ of births in 2007-2008 [2]. Following a caesarean section, it has been reported that $3 \%$ to $15 \%$ of patients develop an SSI [1, 3].

Many studies have examined and reported benchmark post-caesarean section SSI rates, with differing results. For example, the National Healthcare Safety Network (NHSN) conducts a multitude of studies on SSIs using a risk index classification system. The NHSN risk index is defined as either a score of $0,1,2$, or 3, with benchmark rates for postcaesarean section SSIs presenting at $1.46 \% ; 2.43 \%$ for level 0 and 1 , respectively; and $3.82 \%$ for 2 and 3 combined [4]. Additionally, in a study conducted in 14 National Health Service (NHS) hospitals in England, a crude post-caesarean section SSI rate of $9.6 \%$ was reported [5]. These two published studies demonstrate some of the research done on postcaesarean section SSI rates and represent the low and high ends of reported benchmark SSI rates. 
Obstetric infections pose a threat to post-caesarean section patients, as they account for approximately $12 \%$ of maternal deaths [6]. Thus, it is important to monitor the rate of infections within a hospital and to compare the rates of incidence with other benchmarks. Additionally, there was a perceived increase of SSIs in post-partum patients at the community hospital, which provided impetus for this study. This study provides another SSI benchmark that can inform the ongoing understanding of surgical infection rates after caesarean sections.

\section{METHODS}

The purpose of this study was to generate a post-caesarean SSI rate for the community hospital and to compare it to other reported SSI rates. The study population was derived from one community hospital and patients were recruited during a sevenmonth time period. Data was collected from patients who were over the age of 18, who consented to participate in the study, and who underwent a caesarean section between November 2015 and May 2016 at the community hospital.

The research setting was a community hospital that serves a large rural area but is situated in a city of approximately 30,000 residents. The hospital is part of an alliance with three other, smaller community hospitals and provides comprehensive services with 118 beds [7]. The community hospital performs on average 250 caesarean sections annually.

There were two main data collection points. The first consisted of a qualitative survey distributed to the patients' obstetrician-gynecologists (OB-GYNs) for completion at the six-week post-operative follow-up appointment. The second collection period involved a retrospective chart review related to patient demographics and risk factors. Both data sets were collated and quantified for analysis. Demographic data and risk factors gathered in the second collecting period were then used to assign each patient a NHSN risk index factor. A crude SSI rate and NHSN SSI rates, separated by NHSN risk index scores, were calculated. A comparison of the community hospital's postcaesarean section SSI rates with other reported post-caesarean section SSI rates was conducted using statistical evaluations.

The survey distributed to OB-GYNs at the six-week follow-up appointment requested the following information: patient's hospital identification number, surgeon's name, date of the follow-up appointment, and whether the appointment was scheduled or an emergency visit. OB-GYNs also reported if the patient had an SSI and, if so, whether the SSI was classified as either a superficial incisional SSI, a deep incisional SSI, an organ/space SSI, endometritis, no infected complications, or other. Attached to the survey form was a table published by the Centers for Disease Control and Prevention that classifies superficial incisional SSIs, deep incisional SSIs, and organ/space SSIs [8].

Patients' demographic data was retrospectively collected through an analysis of patient medical charts. Factors were identified as important data collection points following a review of related literature and so that NHSN risk indexes could be generated [9-13]. The following factors were recorded: age of patient, gestational age at time of operation, nature of caesarean section (emergency or elective), American Society of Anesthesiologists (ASA) score, wound class, if prophylactic antibiotics were used, if the patient experienced premature rupture of membranes (PROM) or had a predisposing illness, and length of procedure from cut to close.

Following analysis of both the surveys and the medical records, each patient's risk factor was determined using the NHSN classification method. One point was assigned for each of the following indicators: an ASA score between 3 and 5, a wound classification of dirty or contaminated, and/or an operation that lasted longer than the recommended average length for the procedure [14]. A target length of 56 minutes for the caesarean section was chosen, as this is the duration recommended in the NHSN report [4]. In accordance with the NHSN process, each of these categories were allocated a rating of 1 , and patients were then ranked with a composite risk score [4].

Once data was collated, the crude SSI rate and the NHSN risk-adjusted SSI rates were calculated. The crude SSI rate was calculated using the following formula: [total number of infections/total number of caesarean sections performed] $x$ 100. The NHSN risk indexes that were assigned to each patient were used to calculate the NHSN risk-adjusted SSI rate. Each risk-adjusted SSI rate was calculated using the following formula: [number of infections in each risk index/number of caesarean sections performed in each risk index] x 100 [15]. In order to compare the community hospital's SSI rate with other benchmarks, various other studies were selected from the literature. Statistical analysis of compared benchmark rates were calculated using MedCalc and the "N-1" chi-squared test, as recommended by Campbell (2007) [16] and Richardson (2011) [17]. The confidence interval was calculated according to the recommended method given by Altman et al. (2000) [18].

Standard of care following the caesarean section was not altered for patients participating in this study, as a six-week post-operative follow-up appointment is the recommended practice. If a patient developed a complication before the follow-up visit, they were advised to contact the surgeon's office. If the patients were seen at the Emergency department or family physician's office, physicians were advised to contact the patients' surgeons so that data could be accurately recorded. The study protocol was approved by the University of Western Ontario and Huron Perth Healthcare Alliance ethics review boards.

\section{RESULTS}

The study population was derived from one community hospital and patients were recruited during a seven-month time period. A total of 123 patients consented at the outset to participate in the study; however, survey data was not received for five patients. Therefore, the results of this study represent the 118 patients who underwent caesarean sections in the time period and for whom surveys were collected. The patients ranged in age from 20 to 45 years old. The majority (87.3\%) delivered at the gestational age of between 35 and 39 weeks. 75 of the 118 procedures were noted as emergency procedures (63.6\%). 
In addition to demographic information, a review of predisposing illnesses and risk factors was conducted. The highest reported risk factor in the study population was gestational diabetes, wherein $8.5 \%$, or ten patients, had this diagnosis. Three patients were reported to have had gestational hypertension and one patient was reported to have had diabetes. Body mass index (BMI) was only recorded for patients with a BMI above 40 , and those patients with scores between 40 and 50 represented $2.5 \%$ of the overall study population, or three patients. One patient had a recorded BMI above 50 .

Table 1 depicts data that was used to calculate the NHSN risk index scores for the cohort patient population $[4,14]$.

Table 2 reports the crude SSI rate and a risk-adjusted SSI rate for each risk index level [4].

The following observations can be made related to specific demographic and operational data of the seven patients who developed SSIs. Of particular note is the fact that the majority of the patients, or five patients in the cohort of those infected, had developed an SSI following an emergency caesarean section. Additionally, although there are four cases of predisposing illnesses, including gestational diabetes, PROM, an elevated BMI, and/or diabetes, some patients had more than one predisposing illness. This information will not be broken out in order to maintain patient confidentiality.

\section{DISCUSSION}

The purpose of this study was to critically examine the crude and risk-adjusted SSI rates after caesarean sections at a community hospital and to compare these rates with other benchmarks. Studies from Nova Scotia, Saskatchewan, England, and Texas were chosen as basis for crude rate comparison. Statistical analysis was conducted to compare each of the chosen four benchmarks with the community hospital's SSI rate.

The Nova Scotia study consisted of 25,123 patients over a 16-year period and reported a crude SSI rate of 2.7\% [19]. Statistical analysis of this study compared to the community hospital's SSI rate shows a difference of $3.2 \%$, a $95 \%$ confidence interval $(\mathrm{Cl})$ of 0.1747 to 9.0023 , a chi-squared value of 4.555 , degrees of freedom (DF) of 1 , and a significance level of $p=0.0328$ [20]. Saskatchewan performed an evaluation of eight regional authorities with a combined cohort of 3,437 patients over a one-year period with a reported crude SSI rate of $3.5 \%$ [15]. Statistical analysis showed a difference of $2.4 \%$, a $95 \% \mathrm{Cl}$ of -0.6915 to 8.2266 , a chi-squared value of 1.903, DF of 1 , and a significance level of $p=0.1677$ [20]. A third study from England collated a study cohort of 4,107 patients from $14 \mathrm{NHS}$ hospitals over a six-month period and demonstrated a crude SSI rate of 9.6\% [5]. Statistical analysis of this study compared to the community hospital showed

\begin{tabular}{|c|c|c|c|c|}
\hline \multirow[b]{2}{*}{ Characteristic } & \multirow{2}{*}{\multicolumn{2}{|c|}{$\begin{array}{c}\text { Number of Patients } \\
\text { SSI developed }\end{array}$}} & \multirow{2}{*}{\multicolumn{2}{|c|}{$\begin{array}{c}\text { Percentage of Patients } \\
\text { SSI developed } \\
\end{array}$}} \\
\hline & & & & \\
\hline $\begin{array}{l}\text { Wound class } \\
\text { Clean } \\
\text { Clean-contaminated } \\
\text { Contaminated } \\
\text { Dirty } \\
\text { Not reported }\end{array}$ & $\begin{array}{r}31 \\
78 \\
0 \\
0 \\
2 \\
\end{array}$ & $\begin{array}{l}2 \\
5 \\
0 \\
0 \\
0\end{array}$ & $\begin{array}{r}27.9 \% \\
70.3 \% \\
0.0 \% \\
0.0 \% \\
1.8 \% \\
\end{array}$ & $\begin{array}{r}28.6 \% \\
71.4 \% \\
0.0 \% \\
0.0 \% \\
0.0 \% \\
\end{array}$ \\
\hline $\begin{array}{l}\text { ASA score } \\
\text { ASA } 1 \\
\text { ASA } 2 \\
\text { ASA } 3 \\
\text { ASA } 4 \\
\text { ASA } 5 \\
\text { ASA } 6\end{array}$ & $\begin{array}{r}40 \\
39 \\
31 \\
1 \\
0 \\
0\end{array}$ & $\begin{array}{l}2 \\
3 \\
2 \\
0 \\
0 \\
0\end{array}$ & $\begin{array}{r}36.0 \% \\
35.2 \% \\
27.9 \% \\
0.9 \% \\
0.0 \% \\
0.0 \%\end{array}$ & $\begin{array}{r}28.6 \% \\
42.8 \% \\
28.6 \% \\
0.0 \% \\
0.0 \% \\
0.0 \%\end{array}$ \\
\hline $\begin{array}{l}\text { Length of procedure } \\
<56 \text { minutes } \\
\geq 56 \text { minutes }\end{array}$ & $\begin{array}{r}106 \\
5\end{array}$ & $\begin{array}{l}7 \\
0\end{array}$ & $\begin{array}{r}95.5 \% \\
4.5 \%\end{array}$ & $\begin{array}{r}100.0 \% \\
0.0 \%\end{array}$ \\
\hline
\end{tabular}

TABLE 2: Crude SSI rate vs NHSN risk index-adjusted SSI rate.

\begin{tabular}{|l|c|c|c|}
\hline Parameter & Number of Procedures & Number of Infections & Surgical Site Infection Rate \\
\hline Crude SSI calculations & 118 & 7 & $5.9 \%$ \\
\hline NHSN risk-adjusted SSI calculations & & 5 & \\
Risk index 0 & 82 & 2 & $6.1 \%$ \\
Risk index 1 & 34 & 0 & $5.9 \%$ \\
Risk index 2 & 2 & 0 & $0.0 \%$ \\
Risk index 3 & 0 & $0.0 \%$ \\
\hline
\end{tabular}


a difference of $3.7 \%$, a $95 \% \mathrm{Cl}$ of -2.1631 to 6.8611 , a chisquared value of 1.827, DF of 1 , and a significance level of $p=0.1765$ [20]. The last study used for comparison was from Texas. It comprised a review of 19 hospitals, representing 57,182 patients over a three-year period, and reported a crude SSI rate of $6.5 \%$ [21]. Statistical analysis for comparison shows a difference of $0.6 \%$, a $95 \% \mathrm{Cl}$ of -5.2026 to 3.6253 , a chisquared value of 0.070 , DF of 1 , and a significance level of $p=0.7917$ [20]. The community hospital's crude benchmark SSI rate of $5.9 \%$ lands in the middle of the range of other benchmark SSI ratings.

Studies were selected from both Saskatchewan and the NHSN as they offer benchmarks using the risk-adjusted SSI rates. As noted in Results, the community hospital reports an SSI rate for risk index 0 as $6.1 \%$ (82 procedures), for risk index 1 as 5.9\% (34 procedures), and for risk index 2 and 3 as $0.0 \%$ (two procedures). The NHSN study found risk level 0 had an SSI rate of $1.46 \%$ (20,743 procedures) [4]. Statistically, this compared to the community hospital's findings with a difference value of $4.64 \%$, a $95 \% \mathrm{Cl}$ of 1.1696 to 12.0368 , a chi-squared value of 12.073 , DF of 1 , and a significance level of $p=0.0005$ [20]. NHSN risk level 1 had an SSI rate of $2.43 \%$ (8,995 procedures); statistical values were calculated with a difference value of $3.47 \%$, a $95 \% \mathrm{Cl}$ of -0.8075 to 16.6910, a chi-squared of 1.711, DF of 1 , and a significance level of $p=0.1909$ [4, 19]. Risk indexes 2 and 3 from the NHSN were reported with a combined SSI rate of 3.82\% (1,256 procedures) [4]. Comparative statistical analysis could not be completed, as the null hypothesis was not comparable. In the same Saskatchewan study mentioned above, patients were categorized into NHSN risk indexes and SSI rates were calculated for each NHSN risk index. For risk index 0, an SSI rate of $2.8 \%$ was reported (2,496 procedures) [15]. Statistical analysis comparing to the community hospital's results show a difference of $3.3 \%$, a $95 \% \mathrm{Cl}$ of -0.2408 to 10.7178 , a chisquared value of 3.064, DF of 1 , and a significance level of $p$ $=0.0800$ [20]. Risk index 1 reported an SSI rate of 5.1\% (692 procedures), with a comparative statistical analysis of difference of $0.8 \%$, a $95 \% \mathrm{Cl}$ of -3.8686 to 14.0921 , a chi-squared value of 0.042 , DF of 1 , and a significance value of $p=0.8367[15,19]$. The Saskatchewan study reported an SSI rate for risk index 2 and 3 of $14.0 \%$ (50 procedures) [15]. No statistical analysis was calculated, as null hypothesis is not comparable. Not included in the Saskatchewan study NHSN risk index breakdown are 162 procedures with unknown risk indexes with a $4.3 \%$ calculated SSI rate [15].

Both of these study findings demonstrate increasing SSI rates as risk indexes increase. The studies' findings would support the supposition that increased risk indexes would contribute to an increased likelihood of the development of an SSI postcaesarean section [4]. This result differs from the findings of the present study, where a higher SSI incidence rate was found in those patients identified with the lowest risk level. These differences could be attributed to the limited number of patients (118) in the study cohort and, even more significantly, to the small number of patients (seven) presenting with an SSI.
It is expected that results could differ if this study duration was extended and therefore would be able to access a larger cohort of participants.

Nonetheless, a noteworthy finding is the prevalence of emergency caesarean sections for patients in this study. At $63.6 \%$ (75 procedures), most of the caesarean sections were performed as emergency procedures. Multiple studies have concluded that the performance of an emergency caesarean section increases the risk of the development of an SSI, and this could have contributed to the elevated rate of SSIs for those who appeared to have low risk factors $[22,23]$. In this study, five of the seven patients (71.4\%) presenting with an SSI had undergone emergency caesarean sections.

This study faced limitations, first and foremost related to the size of the study cohort. With only seven reported SSIs, the study was limited in its ability to generalize findings. Additionally, given that part of this study was premised upon predominantly qualitative information provided through surveys completed by numerous OB-GYNs, there could also be questions about the reliability and validity of the data, as well as limitations reflected in the physicians' willingness to complete the surveys. Recall that five surveys from consenting participants were not received for analysis. Also, patients who presented to other physicians with normal wound swelling and inflammation could have been misdiagnosed with an SSI. Therefore, there is the possibility that some data could represent false positives. And, finally, there were limitations in the data collection processes in that information about some risk factors, such as obesity, were not comprehensively collected. Specifically, while a BMI above 35 is identified as obese, the surveys only collated data for those patients with levels above 40. Studies would identify obesity as a predictive risk factor and a fulsome collection of this information for this study could have provided another lens through which to examine the results $[6,24]$.

Despite the limitations of the cohort size and data collection mechanisms, this study adds value by contributing to the literature on SSI rates and provides opportunities for next steps. The methodology is replicable and could serve as a means for this or other community hospitals to develop quality control indicators. Moreover, there are advantages that resulted from our methodology. These include improved classification of SSIs through physician diagnosis and reporting of these infections. Furthermore, by advising all physicians to refer patients with suspected SSIs to OB-GYNs, both patient records and SSI information were collated into the centralized hospital database.

Additionally, this study could support the development of a provincial SSI surveillance program with established benchmark targets similar to those established in Nova Scotia and Saskatchewan $[15,20]$. A provincial SSI benchmark would enable hospitals to have evidence-informed comparatives, which could then be monitored and addressed. An increased understanding of the SSI rates for caesarean sections across the province could inform decisions about how to best minimize patient risks and decrease complications related to this increasingly prevalent procedure. 


\section{REFERENCES}

1. Saeed, K. B. M., Greene, R. A., Corcoran, P., \& $\mathrm{O}^{\prime}$ Neill, S. M. (2017). Incidence of surgical site infection following caesarean section: A systematic review and meta-analysis protocol. BMJ Open, 7(1), e013037. Retrieved from http://doi.org/10.1136/ bmjopen-2016-013037

2. Canadian Institute for Health Information. (2018). C-section rates continue to increase while birth rates decline. Retrieved from https:// www.cihi.ca/en/c-section-rates-continue-to-increase-while-birthrates-decline?utm_source $=$ crm\&utm_medium $=$ emailmedemb\&utm campaign $=$ hospch $\&$ utm_content $=$ mediareleaseEN

3. Schneid-Kofman, N., Sheiner, E., Levy, A., \& Holcberg, G. (2005). Risk factors for wound infection following cesarean deliveries. International Journal of Gynecology \& Obstetrics, 90(1), 10-15. doi: 10.1016/j. ijgo.2005.03.020

4. Edwards, J. R., Peterson, K. D., Mu, Y., Banerjee, S., Allen-Bridson, K., Morrell, G., Dudeck, M. A., Pollock, D. A., \& Horan, T. C. (2009). National Healthcare Safety Network (NHSN) report: Data summary for 2006 through 2008, issued December 2009. American Journal of Infection Control, 37(10), 783-805. doi: 10.1016/j.ajic.2009.10.001

5. Wloch, C., Wilson, J., Lamagni, T., Harrington, P., Charlett, A., \& Sheridan, E. (2012). Risk factors for surgical site infection following caesarean section in England: Results from a multicentre cohort study. BJOG: An International Journal of Obstetrics \& Gynaecology, 119(11), 1324-1333. doi: https://doi.org/10.1111/j.1471-0528.2012.03452.x

6. Witter, F. R., Lawson, P., \& Ferrell, J. (2014). Decreasing cesarean section surgical site infection: An ongoing comprehensive quality improvement program. American Journal of Infection Control, 42(4), 429-431. doi: https://doi.org/10.1016/j.ajic.2013.12.004

7. SouthWest Healthline. (2018). Stratford General Hospital. Retrieved from https://www.southwesthealthline.ca/displayservice.aspx?id=15284

8. Centers for Disease Control and Prevention. (2018). Surgical site infection (SSI) event. Retrieved from https://www.cdc.gov/nhsn/PDFs/ pscManual/9pscSSlcurrent.pdf

9. Bianco, A., Roccia, S., Nobile, C. G., Pileggi, C., \& Pavia, M. (2013) Postdischarge surveillance following delivery: The incidence of infections and associated factors. American Journal of Infection Control, 41(6), 549-553. doi: 10.1016/j.ajic.2012.06.011

10. Wilson, J., Wloch, C., Saei, A., McDougall, C., Harrington, P., Charlett, A., Lamagni, T., Elgohari, S., \& Sheridan, E. (2013). Inter-hospital comparison of rates of surgical site infection following caesarean section delivery: Evaluation of a multicentre surveillance study. Journal of Hospital Infection, 84(1), 44-51. doi: 10.1016/j.jhin.2013.01.009

11. Smaill, F. M., \& Grivell, R. M. (2014). Antibiotic prophylaxis versus no prophylaxis for preventing infection after cesarean section. Cochrane Database of Systematic Reviews, (10), CD007482. doi: 10.1002/14651858.CD007482.pub3

12. Ng, W., Brown, A., Alexander, D., Ho, M. F., Kerr, B., Amato, M., \& Katz, K. (2015). A multifaceted prevention program to reduce infection after cesarean section: Interventions assessed using an intensive postdischarge surveillance system. American Journal of Infection Control, 43(8), 805-809. doi: 10.1016/j.ajic.2015.04.001

13. Moulton, L. J., Munoz, J. L., Lachiewicz, M., Liu, X., \& Goje, O. (2018). Surgical site infection after cesarean delivery: Incidence and risk factors at a US academic institution. Journal of Maternal-Fetal \& Neonatal Medicine, 31(14), 1873-1880. doi: 10.1080/14767058.2017.1330882

14. Provincial Infectious Diseases Advisory Committee. (2014). Best practices for surveillance of healthcare-associated infections. Retrieved from https://www.publichealthontario.ca/en/eRepository/ Surveillance_3-3_ENGLISH_2011-0-28\%20FINAL.pdf

15. Government of Saskatchewan. (2016). Saskatchewan caesarean section surgical site infection surveillance report: 2015-16. Retrieved from https://www.ehealthsask.ca/services/resources/Resources/SSI\%20 Surveillance\%20Annual\%20Report\%202015-16.pdf

16. Campbell, I. (2007). Chi-squared and Fisher-Irwin tests of two-by-two tables with small sample recommendations. Statistics in Medicine, 26(19), 3661-3675. doi: 10.1002/sim.2832
17. Richardson, J. T. (2011). The analysis of $2 \times 2$ contingency tables-yet again. Statistics in Medicine, 30(8), 890. doi: 10.1002/sim.4116

18. Altman, D. G., Machin, D., Bryant, T. N., \& Gardner, M. J. (Eds.). (2000). Statistics with confidence: Confidence intervals and statistical guidelines (2nd ed.). London, UK: BMJ Books.

19. Ketcheson, F., Woolcott, C., Allen, V., \& Langley, J. M. (2017). Risk factors for surgical site infection following cesarean delivery: A retrospective cohort study. CMAJ Open, 5(3), E546-E556. doi: 10.9778/ cmajo.20160164

20. MedCalc. (2018). Comparison of proportions calculator. Retrieved from https://www.medcalc.org/calc/comparison_of_proportions.php

21. Haidar, Z. A., Nasab, S. H., Moussa, H. N., Sibai, B. M., \& Blackwell, S. M. (2018). Caesarean delivery surgical site infection: What are expected rates and potentially modifiable risk factors? Journal of Obstetrics and Gynaecology Canada, 40(6), 684-689. doi: https://doi.org/10.1016/j. jogc.2017.09.020

22. Farret, T. C. F., Dallé, J., Monteiro, V. da S., Riche, C. V. W., \& Antonello, V. S. (2015). Risk factors for surgical site infection following cesarean section in a Brazilian women's hospital: A case-control study. Brazilian Journal of Infectious Diseases, 19(2), 113-117. doi: https://doi. org/10.1016/j.bjid.2014.09.009

23. Alishaq, M. A., AlAjmi, J. A., Al-Ali, B., Saleh, F., El-Sheik, M., Malkawi, M., George, L., Garcia, L., \& Locus, B. (2011). Risk factors of surgical site infection post cesarean section. BMC Proceedings, 5(Suppl 6), 189. doi: http://doi.org/10.1186/1753-6561-5-S6-P189

24. Kawakita, T., \& Landy, H. J. (2017). Surgical site infections after cesarean delivery: Epidemiology, prevention and treatment. Maternal Health, Neonatology and Perinatology, 3, 12. doi: http://doi.org/10.1186/ s40748-017-0051-3 